\title{
In phase with synthesis
}

\author{
The field of organic synthesis has benefited from a greater understanding of organometallic and coordination \\ chemistry, and the applications of homogeneous catalysts continue to impress.
}

T he history of organic synthesis and catalysis are so closely entwined that it can be difficult to pick them apart. And when it comes to the synthesis of fine chemicals, homogeneous systems in particular have had pride of place in the chemist's toolkit. Simple Brønsted acids may represent the bulk of such catalysts, and indeed the first applications of non-enzymatic catalysis in synthesis relied on these. As early as the midsixteenth century Valerius Cordus reported the sulfuric-acid-catalysed synthesis of diethyl ether from ethanol ${ }^{1}$, a process still taught in textbooks to this day. The observation by Antoine-Augustin Parmentier that acetic acid could speed up the conversion of starch into a sweet substance - now known to be glucose - was, in hindsight, another major milestone in the development of homogeneous catalysis (though outside the world of catalysis he will more likely be remembered for determined and single-minded promotion of potatoes as a foodstuff) ${ }^{2}$.

But going beyond Brønsted acids, metal complexes are probably what most people imagine when thinking about homogeneous catalysis. And for this field to reach its potential, a necessary step was the development and understanding of coordination and organometallic chemistry. As the mastery of metal complexes increased, so too did the dizzying numbers of applications available to the synthetic chemist: the discoveries - and the Nobel Prizes - kept rolling in.

Of special note is the development of asymmetric catalysis. The initial success of catalytic asymmetric hydrogenation by Knowles ${ }^{3}$ was a direct result of the earlier work by Wilkinson on a soluble hydrogenation catalyst, the synthesis of which would not have been possible without earlier and more fundamental work on metal complexes. And the enantioselectivities achieved by these new molecular catalysts were useful - showing that this was a more promising avenue to explore than earlier attempts to insert metals into a chiral heterogeneous support ${ }^{4}$. Subsequently, the work of Sharpless ${ }^{5}$ on asymmetric oxidations showed that useful enantioselectivites were not limited to hydrogenation reactions.

Another key development for organic synthesis - and another Nobel Prize - came with the use of palladium for cross-coupling. The ability to form carbon-carbon bonds (especially those involving $s p^{2}$ carbons) with ease has led to these methods becoming a workhorse for the synthetic chemist. However, given the cost and toxicity of palladium compounds it is unsurprising that significant efforts in cross-coupling reactions - and indeed catalysis in general have been devoted to developing analogous reactivity with earth-abundant metals. And over the years we have seen reaction after reaction that were previously the domain of rare and precious metals fall under a wave of inexpensive salts. Iron in particular has been the focus of determined efforts, spurred on by its abundance and low cost. And this is not a new trend: it is worth noting that as far back as 1971 Tamura and Kochi could open their report ${ }^{6}$ with the statement that "iron is representative of one of the most effective metal catalysts for the promotion of the reactions between Grignard reagents and organic halides". And the progress has been sufficiently impressive that in a recent review ${ }^{7}$ Bauer and Knölker accurately state that "it appears now that iron-catalysed reactions cover almost the full scope of transformations which are presented in organic textbooks". In the context of so much success, it is interesting to note the cases where iron has struggled to replicate the behaviour of palladium. Perhaps surprisingly, one such difficulty has been presented by the simple Suzuki biaryl cross-coupling, with very limited, and not generally applicable, solutions reported so far. In this issue, Robin Bedford and co-workers provide a method that fills this gap, as they report iron-catalysed coupling of aryl halides and boronates under mild conditions with a surprisingly large scope. The key is the use of an $N$-pyrrole amide as a directing group and, interestingly, mechanistic studies indicate that this binds to the metal through $\pi$-interactions. The directing group is needed for the initial activation of the carbon-halide bond, while the remainder of the steps in the catalytic cycle are easier - consistent with the idea that it is the difficult $\mathrm{C}-\mathrm{X}$ activation that has presented the bottleneck in this procedure.

\section{Looking forward, it seems certain that the study of orga- nometallics has a lot more to offer to organic synthesis.}

Next up in the issue is Pher Andersson and co-workers reporting a dynamic kinetic resolution of secondary allylic alcohols. One obvious strength of this method is the range of products - an array of alcohols with two contiguous stereocentres are produced with enantiomeric excesses up to $99 \%$. Beyond that, the reaction itself is intriguing. To achieve dynamic kinetic resolution it is necessary to racemize the starting material, which in this case contains a stereocentre adjacent to the hydroxyl group. The authors make a good case that the resolution process proceeds via cleavage of the carbon-oxygen bond.

Another interesting report in this issue takes a new look at Kolbe-Schmidt type reactions. This venerable method — dating from 1860 as a means for producing salicylic acid ${ }^{8}-$ allows for carboxylations of aryl C-H bonds with high pressures of $\mathrm{CO}_{2}$. Here Gang $\mathrm{Li}$ and co-workers show that for 2-(hetero) arylphenols, an appropriate metal/ligand combination makes it possible to direct the carboxylation to the less nucleophilic aromatic ring, using atmospheric pressures of $\mathrm{CO}_{2}$.

Looking forward, it seems certain that the study of organometallics has a lot more to offer to organic synthesis. Even as chemists develop computational tools to aid them in their synthesis design ${ }^{9,10}$, they will still need new transformations and economically viable catalysts to work with, especially as the targets become more complex. New methods and new uses for underused metals will always be in demand.

Published online: 12 June 2018

https://doi.org/10.1038/s41929-018-0103-8

References

1. Leake, C. D. Isis 7, 14-24 (1925).

2. Zecchina, A. \& Califano, C. The Development of Catalysis: A

History of Key Processes and Personas in Catalytic Science and Technology (Wiley, Hoboken, 2017).

3. Knowles, W. S. Angew. Chem. Int. Ed. 41, 1998-2007 (2002).

4. Akabori, S., Sakurai, S., Izumi, Y. \& Fujii, Y. Nature 178, 323-324 (1956).

5. Sharpless, B. Angew. Chem. Int. Ed. 41, 2024-2032 (2002).

6. Tamura, M. \& Kochi, J. K. J. Am. Chem. Soc. 93 , 1487-1489 (1971)

7. Bauer, I. \& Knölker, H.-J. Chem. Rev. 115, 3170-3387 (2015).

8. Kolbe, H. Justus Liebigs Ann. Chem. 113, 125-127 (1860).

9. Segler, M. H. S., Preuss, M. \& Waller, M. P. Nature 555 , 604-610 (2018).

10. Klucznik, T. et al. Chem 4, 522-532 (2018). 